\title{
La planeación financiera como herramienta de gestión para mejorar la rentabilidad en las instituciones financieras
}

Financial planning as a management tool to improve profitability in financial institutions

Marco Vinicio Salazar Tenelanda. ${ }^{1}$, Paulina Fernanda Alvear Haro. ${ }^{2}$ \& Myriam Cecilia Sampedro Redrobán. ${ }^{3}$

Recibido: 10-02-2019 / Revisado: 15-02-209 /Aceptado: 04-03-2019/ Publicado: 05-04-2019

\begin{abstract}
.
DOI: https://doi.org/10.33262/cienciadigital.v3i2.487

The development of this research was aimed at determining financial strategies that allow improving the profitability of a financial institution, promoting the placement of credit in the medium and long term, to stimulate performance in the productive management of the business sector. After identifying the problem, this study made a diagnosis based on surveys, interviews and financial analyzes of the bank's balance sheets, which helped define the strategies, established as tools to improve the bank's profitability. It was demonstrated with the results obtained that the clients of the entity under study are dissatisfied with the credit conditions currently established, that the profitability in general is low, obtaining an ROE of $5.6 \%$ and a ROA of $0.49 \%$. It is concluded that an adequate financial diagnosis is fundamental for decision making, and at the same time it is recommended to apply the proposed strategies which according to the financial performance and applying the profitability indicators to the projected balance sheets, an ROE of $7.15 \%$ was obtained. and an ROA of $0.67 \%$ showing that it is necessary to change strategies and policies in the placement and capture of resources in the bank under study, in order to get out in the highly competitive financial market.
\end{abstract}

\footnotetext{
${ }^{1}$ Escuela Superior Politécnica de Chimborazo, Facultad de Administración de Empresas, Riobamba, Ecuador, salazarmarco1977@gmail.com

${ }^{2}$ Escuela Superior Politécnica de Chimborazo, Facultad de Administración de Empresas, Riobamba, Ecuador, paulina.alvear@espoch.edu.ec

${ }^{3}$ Escuela Superior Politécnica de Chimborazo, Facultad de Administración de Empresas, Riobamba, Ecuador, myriam.sampedro@espoch.edu.ec
} 
Keywords: Planning, Management, Financial, Strategies, Profitability, Bank.

\section{Resumen.}

El desarrollo de esta investigación tuvo como objetivo determinar estrategias financieras que permitan mejorar la rentabilidad de una institución financiera, potenciando la colocación del crédito a mediano y largo plazo, para estimular el desempeño en la gestión productiva del sector empresarial. Este estudio luego de identificar la problemática realizó un diagnóstico basado en encuestas, entrevistas y análisis financieros a los balances de la entidad, los mismos que ayudaron a definir las estrategias, establecidas como herramientas para mejorar la rentabilidad del banco. Se demostró con los resultados obtenidos que los clientes de la entidad en estudio están inconformes con las condiciones del crédito establecidas actualmente, que la rentabilidad en general es baja, obteniendo un ROE del 5,6\% y un ROA del 0,49\%. Se concluye que un adecuado diagnóstico financiero es fundamental para la toma de decisiones, y a la vez se recomienda aplicar las estrategias propuestas las cuales según la corrida financiera y aplicando los indicadores de rentabilidad a los balances proyectados, se obtuvo un ROE del 7,15\% y un ROA del 0,67\% evidenciando que es necesario el cambio de estrategias y políticas en la colocación y captación de recursos en el banco en estudio, para de esta manera sobre salir en el mercado financiero altamente competitivo.

Palabras claves: Planeación, Gestión, Financiera, Estrategias, Rentabilidad, Banco.

\section{Introducción.}

Para el desarrollo de esta investigación es de suma importancia tener presente que la planeación financiera de acuerdo con la concepción (Correa, Ramirez, \& Castaño, 2010) es:

Una herramienta empresarial que permite la visualización del proyecto empresarial bajo un enfoque global, teniendo en cuenta los diferentes escenarios en donde puede incursionar y los distintos factores que lo impactan. Dicho en otras palabras, este proceso, es el que permite traducir a términos cuantitativos es decir a términos financieros todas las políticas, prácticas y estrategias contempladas en el plan de negocios mediante la modelación financiera, la cual permite la realización de proyecciones y estimaciones financieras, imprimiéndole un carácter estratégico que apunta a la adecuada toma de decisiones (Correa, Ramirez, \& Castaño, 2010).

Es por ello que el presente estudio pretende llevar a cabo un análisis que contribuya internamente al Banco, para tener conocimiento de los beneficios que aporta el plan financiero con la promoción, colocación y recuperación de créditos para la reactivación de la economía y además los beneficios que conlleva el otorgar créditos productivos para las empresas, de esta manera se logrará poseer una herramienta que sirva de base, con 
lineamientos específicos para la toma de decisiones y un mejor desenvolvimiento de los ejecutivos y el personal encargado de esta área; además se podrá obtener una evidencia de las utilidades que se obtendrán después de los cambios y correctivos pertinentes dentro de la planeación financiera.

De cierta manera se verán beneficiados de una manera indirecta los clientes del Banco, debido a que, si la herramienta es implementada, esta tendrá efectos positivos pues al haber una mejora en el manejo y planificación financiera de la institución será evidente la aplicación de estrategias que estén en pro de ayuda y beneficio hacia los clientes.

En este contexto con la presente investigación, se determinó estrategias financieras que permitan mejorar la rentabilidad de una institución financiera, potenciando la colocación del crédito a mediano y largo plazo, para estimular el desempeño en la gestión productiva del sector empresarial, pues este sector es la principal fuente de crecimiento económico que posee el país. Es imperativo tomar acciones sobre las estrategias empleadas para otorgar financiamiento ya que ambas partes se verán beneficiadas, por un lado el sector empresarial así como el banco, que podrá lograr rentabilidad en dichos créditos.

\section{Desarrollo}

\section{Finanzas}

Las finanzas conciernen a una materia de la economía que trabaja en la circulación del capital entre los individuos, las sociedades o el estado, por otra parte, investiga la adquisición y la distribución del dinero que realizan para lograr sus respectivos objetivos, tomando en cuenta todos los riesgos que ello implica (Corporacion Universitaria del Caribe, 2014).

Las finanzas se refieren a la manera en que los dueños, propietarios, gerentes de una empresa administran el dinero y toman decisiones.

\section{Gestión Financiera}

Es la combinación de distintas operaciones y métodos conectados entre sí, con la intención de emprender, proyectar, administrar, vigilar, de forma eficaz y segura, los recursos de personal, económicos, exactos para el movimiento y progreso de las empresas, fiscalizando inflexiblemente, para adquirir los propósitos planteados.

La gestión financiera tiene por propósito la ejecución del presupuesto de gastos aprobado. Este cumplimiento se efectúa mediante diferentes etapas, iniciándose con la autorización del gasto y terminando con su pago material (Cordova, 2012).

Los instrumentos de la gestión financiera 
La administración financiera de las sociedades necesita del uso de ciertas herramientas para poder ejecutar adecuadamente la dirección de los negocios o capitales económicos. Estos instrumentos son adaptables en los procedimientos productivos, industriales y de servicios, entre otros; y se llevan a cabo e inclusive en nuestra actividad personal. Estas ofrecen estructura, información y recursos para sus decisiones relacionadas con el dinero y de esta manera reducir el temor a la incertidumbre financiera y aumentar la capacidad de tomar decisiones, en lugar de tener que reaccionar continuamente ante las crisis (Cordova, 2012).

\section{Diagnostico financiero}

Es el proceso que comprende recopilación, razonamiento, comparación y estudio de los Estados Financieros básicos y demás información sobre la situación de una empresa, involucra el cálculo y análisis de porcentajes, tasas, tendencias, indicadores y estados financieros complementarios, los mismos que sirven para estimar la destreza financiera y estratégica de la entidad, favoreciendo al administrador, inversionista y acreedor hacia la toma de decisiones acertadas.

La ejecución de un análisis económico en la sociedad es importante para una considerada administración. Tolerará a la compañía buscar adaptadamente sus compromisos financieros, negociar pertinentemente, así como optimizar comercializaciones y beneficios, desenvolviendo el importe de la organización.

Para conseguir un estudio útil, se lo debe desarrollar de forma consecutiva en el ciclo con una plataforma de información segura, y ser conducido por directrices enmendadoras para reparar los desvíos que se encuentre (Valladolid Emprende, 2012).

\section{Planeación Financiera}

' 'La planeación financiera es una técnica que reúne un conjunto de métodos, instrumentos y objetivos con el fin de establecer los pronósticos y las metas económicas y financieras de una empresa, considerando los medios que se tienen y los que se requieren para lograrlo"' (Ortega, 2012)

' 'La planeación financiera es un procedimiento en tres fases para decidir qué acciones se habrán de realizar en el futuro, para lograr los objetivos trazados. Planear lo que se quiere hacer, llevar a la práctica lo planeado y verificar la eficiencia de lo que se hace mediante un presupuesto. La planeación financiera dará a la empresa una coordinación general de funcionamiento. Así mismo reviste gran importancia para el funcionamiento y la supervivencia de la organización". (Ortega, 2012)

'En el proceso de planificación financiera la empresa trata de proyectar en términos monetarios el resultado futuro que desea alcanzar y para esto debe establecer los recursos que necesita para lograr el objetivo. Un plan financiero específica y cuantifica en términos 
monetarios el resultado de cada una de las diferentes alternativas de acuerdo con las estimaciones del entorno que se realicen, así como las necesidades financieras que se pueden derivar de cada política empresarial’”. (Diez de Castro \& López, 2008)

\section{Indicadores financieros}

Los indicadores son los medios más comunes para el desarrollo acertado del análisis financiero, representan aquella relación existente entre las cuentas del balance general y el estado de resultados de una empresa.

Así esta información permite la verificación de las fortalezas o debilidades internas, procurando una intervención por medio de probabilidades y supuestos, por ello los indicadores facilitan la relación de las cuentas otorgando sentido a los datos según sea la necesidad requerida, dentro de los cuales se pueden detallar a:

\section{Liquidez}

Indicador o razón que la empresa requiere para la verificación que posee con respecto a cumplir con las obligaciones que posee a corto plazo, es decir, el grado de dificultad o facilidad para cubrir los pasivos corrientes con los activos en un lapso máximo de un año.

\section{Capital de trabajo}

Indicador que determina la razón corriente de forma cuantitativa, relacionando el activo y los pasivos corrientes, verificando de esta manera las disponibilidades con las que cuenta una empresa a corto plazo.

\section{Endeudamiento}

El endeudamiento es un indicador que permite la medición del grado de participación de los acreedores dentro del financiamiento que la empresa incurre. Por tal motivo, es aquel que determinará el riesgo que corren los acreedores o propietarios y así verificar la factibilidad que repercutiría un endeudamiento.

\section{Rentabilidad}

Denominados también como rendimiento, determinan la efectividad que posee la empresa mediante el control adecuado de los costos y los gastos necesarios para la operatividad permitiendo de esta manera obtener utilidades óptimas sobre las ventas efectuadas (Prieto, 2010).

\section{Estrategias financieras}

Las estrategias financieras hacen referencia a un plan financiero a largo plazo el cual constituye la expresión cuantificada en términos monetarios, sistemáticos e integrados. 
Este plan financiero es fundamental porque representa una recapitulación de todo el transcurso de programación, consintiendo relacionar las insuficiencias de capitales que poseerá la compañía a lo largo del ciclo con las fuentes favorables para su endeudamiento.

El financiamiento de la compañía le corresponderá restructurar su técnica, tomando en cuenta las particularidades estratégicas, en la transformación de inversión, diferenciación pertinente de sus desplazamientos de crédito, autofinanciación transcendental como el plan de transformaciones y desinversiones, propósitos de desarrollo a largo plazo, know-how, entre otros.

Si las oportunidades de endeudamiento superan a las insuficiencias de capitales, la empresa puede recapacitar sobre su plan estratégico para fomentar su desarrollo y fortificar su organización prestamista.

El plan de financiamiento de la compañía tolera, examinar la debilidad de la misma ante posibles imprevistos en su contexto, como una caída del mercado, un alza en los precios, demoras en la entrada en conveniencia de las inversiones y fortificación de los mercados financieros, así también estimar su flexibilidad y desplazamiento de acomodo frente a estas circunstancias y a otras condiciones semejantes.

Las habilidades financieras pueden ser formadas por el conveniente proceso de programación o ser convenientes por la alta administración o por la corporación basándose en atenciones de manejo general o a las limitaciones completas.

El plan financiero conlleva a la evaluación de balances y cuentas de resultados futuros.

Se designa técnica financiera al vinculado de los tres instrumentos o etapas proyectivas siguientes:

- Estados de origen y aplicación de fondos

- Balances proyectados

- Cuentas de resultados proyectadas

Los propósitos financieros a largo plazo constituyen de un esquema minucioso de inversiones, desarticulado por indicaciones contables y por segmentaciones, movimientos o líneas de productos, y un plan de endeudamiento.

\section{Crédito}

Cuando se concede un financiamiento, es primordial tener una buena dirección del dinero, en función de las necesidades que se tenga. En los créditos, por lo tanto, se puede cancelar 
una parte o la totalidad de la deuda en el momento que se considere adecuado y el pago de los intereses asociados al mismo.

Generalmente se recurre a los créditos bancarios cuando es preciso generar mayor liquidez para cubrir un gasto que requiera la empresa. Pueden solicitarse mediante una cuenta o una tarjeta según los mecanismos que maneje la entidad financiera otorgante (Del Valle, 2008).

\section{Evolución de los créditos}

Están varios dictámenes con relación a la verificación de la fecha en que nació el crédito, pues mientras varios autores piensan que no pudo haber existido en la antigüedad, otros si creen que se hayan efectuado ordenamientos de endeudamiento aún antes de la era cristiana (Universidad de Sonora, 2007).

El acceso al crédito y al aseguramiento de las actividades comerciales a nivel internacional son elementos que determinan el funcionamiento de la economía mundial. Entre los años 80 y 90 las labores de comercio internacionales estaban respaldadas por acciones financieras crediticias, que compone un componente primordial en el desenvolvimiento del patrimonio total y por ello, uno de los elementos más significativos es la objetividad de limitaciones o suspensiones en el endeudamiento, en específico en las naciones en progreso (Medina \& Montes, 2010).

En el estado ecuatoriano hoy por hoy posee una extensa lista de establecimientos de microfinanzas que son afines a la política de financiamiento para el progreso en el país. Las sociedades financieras, bancos y cooperativas vienen desde 1879 al crearse el primer establecimiento con estas particularidades en la ciudad de Guayaquil, la "Sociedad de Artesanos Amantes del Progreso", a partir de entonces el desarrollo del sistema financiero ha sido muy dinámico.

Además, considerando el número de instituciones financieras y los montos de crédito otorgados se refleja la importancia del sistema de créditos en el país.

El gobierno ecuatoriano ha desempeñado un papel primordial al promover créditos con varios programas para el fortalecimiento y sostenimiento de las pequeñas y grandes empresa, cofinanciadas por corporaciones mundiales, varias de estas entidades se han implicado como son: el Banco Central, Corporación Financiera Nacional y Banco Nacional de Fomento (Endara, 2004). 
Tipos de créditos

Los servicios más demandados por las empresas son el crédito y el ahorro. Siendo el primero el servicio de mayor demanda. Los Bancos brindan varias tipologías, intentando hacerlos llamativos para los empresarios.

Según la normativa vigente en Ecuador existe los siguientes segmentos de crédito:

- Crédito productivo

$\checkmark$ Productivo corportaivo

$\checkmark$ Productivo empresarial

$\checkmark$ Productivo pymes

- Crédito comercial ordinario

- Crédito comercial prioritario

$\checkmark$ Productivo corportaivo

$\checkmark$ Productivo empresarial

$\checkmark$ Productivo pymes

- Crédito de consumo ordinario

- Crédito de consumo prioritario

- Crédito educativo

- Crédito de vivienda

- Crédito inmobiliario

- Microcrédito

$\checkmark$ Minorista

$\checkmark$ Acumulación simple

$\checkmark$ Acumulación ampliada

- Crédito de inversión pública

\section{Metodología.}

Para el desarrollo de la presente investigación se usó diferentes métodos como el teórico, al igual que el método analítico- sintético para la revisión de la literatura y el histórico lógico mismo que es necesario para abordar el tema de gestión financiera, en lo que tiene que ver a sus aspectos teóricos necesarios para el desarrollo de la presente investigación. También se usó el método dialéctico, inductivo y deductivo ya que mediante la aplicación de estos métodos se realizó el análisis del tema.

Para fundamentar teóricamente el presente trabajo se utilizó la investigación documental, además se aplicó la investigación de campo, con el objetivo de descifrar la cultura desde el punto de vista de sus miembros (comprensión). Luego a partir de datos relacionados con otros hallazgos culturales se desarrollan unas generalizaciones (inducción) que, a su vez, guardan relación con otras culturas y teorías ya existentes (explicación). Se desarrolló esta 
investigación, para conocer la realidad, a través de encuestas, entrevistas y cuestionarios para conocer la situación actual.

La Población en la presente investigación corresponde a la cartera de clientes que recibieron créditos empresariales en el año 2017, a quienes se aplicará una encuesta, y por otro lado los representantes del Banco, a quienes se les aplicó un cuestionario de entrevista.

Para el cálculo de la muestra se consideró la población de 969 personas que recibieron créditos empresariales en el año 2017, y mediante la aplicación de la formula se obtuvo una muestra de 275 encuestas a ser aplicadas.

\section{Resultados.}

1. ¿Considera usted que se debería incrementar los cupos para acceder a los créditos productivos?

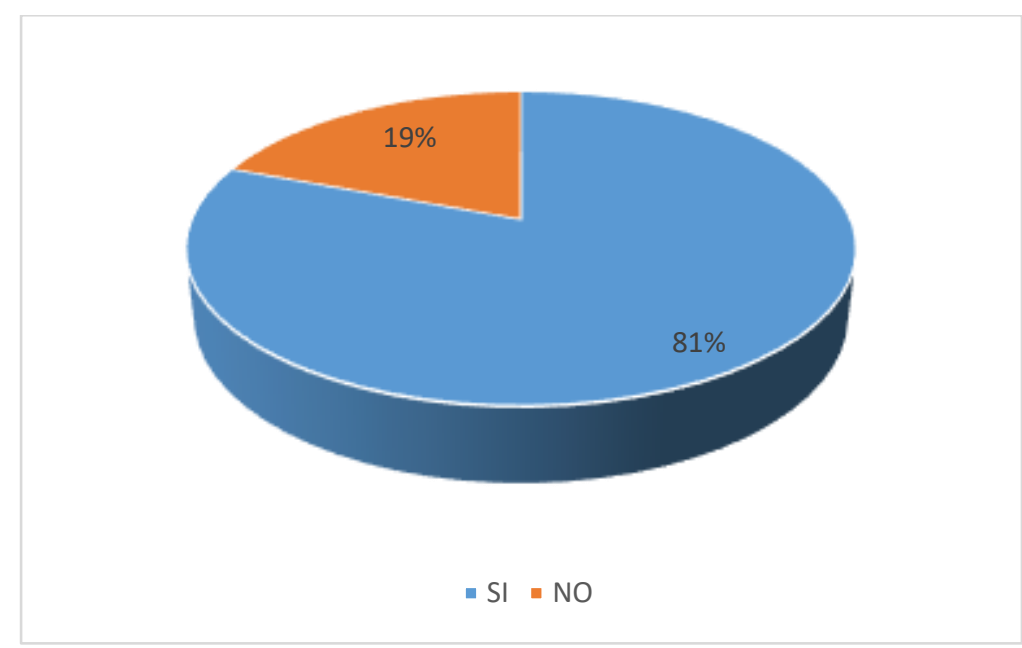

Gráfico $\mathbf{N}^{\circ}$ 1: Incremento de cupos

Fuente: Elaboración propia

Elaborado por: Los autores

\section{Análisis e interpretación}

De acuerdo a los datos obtenidos el $81 \%$ de las personas encuestadas indica que se si están de acuerdo con que la entidad financiera incremente los cupos para acceder a los créditos productivos, mientras que el porcentaje correspondiente al $19 \%$ no.

El Banco debería considerar el incremento de los cupos para el acceso a los créditos productivos con la finalidad de que las pequeñas y medianas empresas fomenten el desarrollo local a través de los préstamos hipotecarios. 
2. ¿Está usted de acuerdo con la tasa de interés del $17 \%$ aplicada por el Banco para el acceso a los créditos productivos?

Tabla 1:Tasa de interés

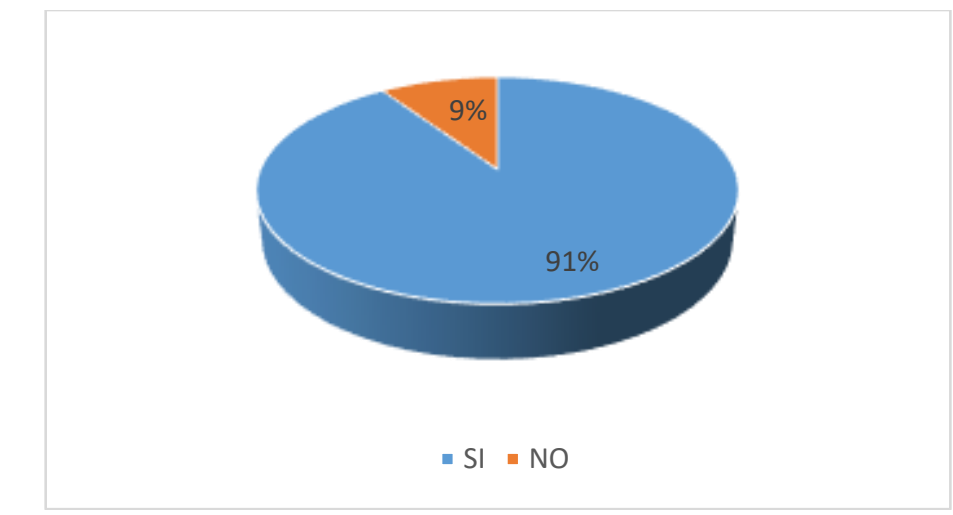

Gráfico $\mathbf{N}^{\circ}$ 2: tasa de interés

Fuente: Elaboración propia

Elaborado por: Los autores

\section{Análisis e interpretación}

Según los datos obtenidos mediante la aplicación de encuestas el $9 \%$ si se encuentra de acuerdo con la tasa de interés que aplica Banco Pichincha para la generación de crédito productivos mientras que el $91 \%$ no se encuentra de acuerdo.

Es necesario considerar la tasa de interés que más se ajuste a las necesidades del sector productivo con la finalidad de que los emprendedores puedan acceder al mismo bajo las necesidades más ajustables para el proceso de pago.

3. ¿Estaría usted de acuerdo con que el banco disminuya los trámites para el acceso a los créditos productivos?

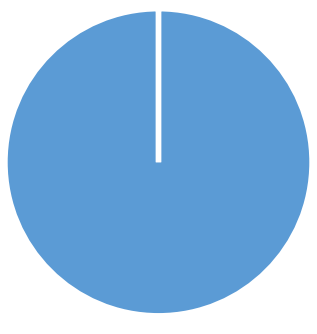

$$
\because \mathrm{SI}=\mathrm{NO}
$$

Gráfico $\mathbf{N}^{\circ}$ 3: trámites

Fuente: Elaboración propia

Elaborado por: Los autores 


\section{Análisis e interpretación}

De acuerdo al gráfico se puede observar que las personas en su totalidad se encuentran de acuerdo con la disminución de trámites para el acceso a los créditos.

Las pequeñas y medianas empresas en su mayor parte requieren del acceso a los créditos para poder incrementar su capital de trabajo sin embargo la generación de trámites hacen que la mayor parte de estas tengan inconvenientes para el acceso al mismo.

\section{4. ¿Cuál es el plazo que usted determina el más adecuado para el acceso al crédito} para capital de trabajo?

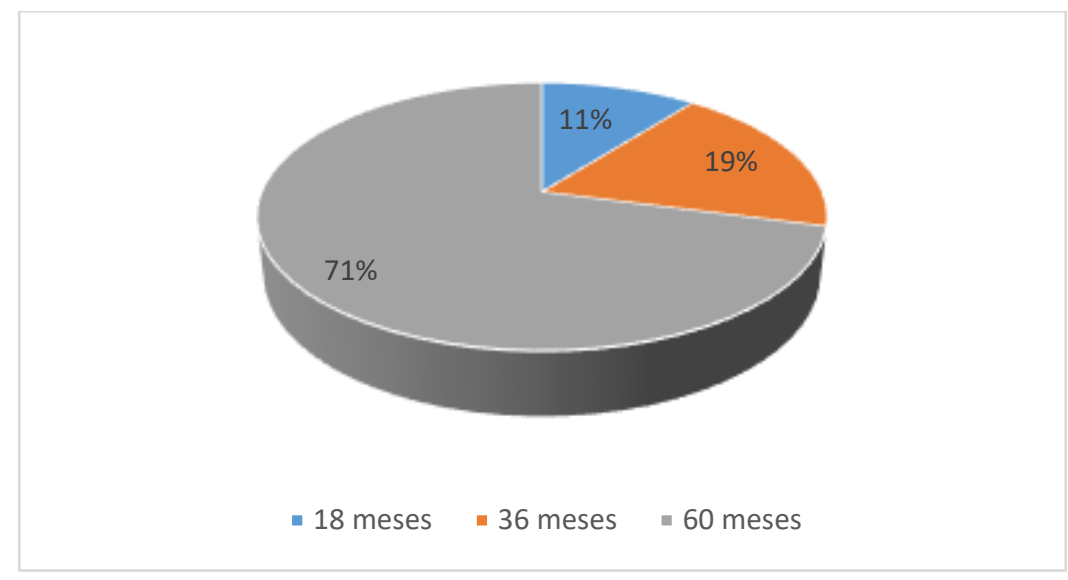

Gráfico $\mathbf{N}^{\circ}$ 4: Plazo para acceso a créditos

Fuente: Elaboración propia

Elaborado por: Los autores

\section{Análisis e interpretación}

De las encuestas realizadas el $71 \%$ en su mayor parte indican que el plazo más accesible ante el acceso a créditos para el capital de trabajo es de 60 meses, mientras que un 19\% considera 36 meses y un mínimo del $11 \%$ establece 18 meses.

De acuerdo a las encuestas el plazo más accesible para los pequeños empresarios para la otorgación de créditos sobre el capital de trabajo es de 60 meses debido a que tienen mayores posibilidades de pagar puntualmente los créditos. 
5. ¿Está usted de acuerdo con la atención que recibe en el banco al momento de recibir los créditos?

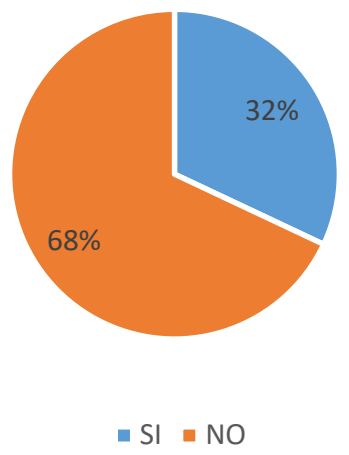

Gráfico $\mathbf{N}^{\circ}$ 5: Atención al cliente

Fuente: Elaboración propia

Elaborado por: Los autores

\section{Análisis e interpretación}

Partiendo de la información obtenida de las encuestas realizadas en su mayor parte $68 \%$ no se encuentra de acuerdo con la atención recibida al momento de la otorgación de los créditos, mientras que un mínimo de $32 \%$ si se encuentra de acuerdo.

Es necesario que la entidad financiera establezca una estrategia que permita mejorar la atención al cliente a la hora de realizar los préstamos considerando las necesidades para un servicio de calidad.

6. ¿Consideraría usted que el Banco debe mejorar las condiciones de los créditos productivos para estimular la gestión financiera de los clientes empresariales?

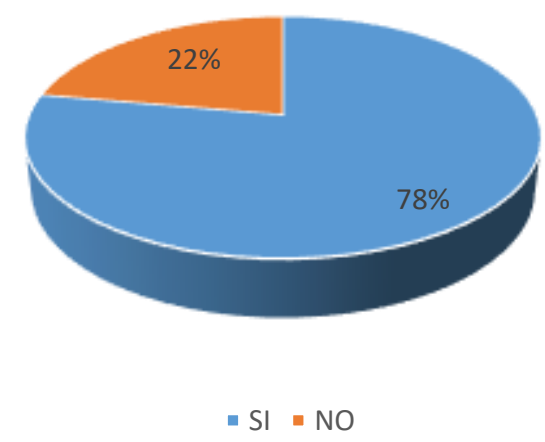

Gráfico $\mathbf{N}^{\circ}$ 6: Mejora de los créditos empresariales Fuente: Elaboración propia

Elaborado por: Los autores 


\section{Análisis e interpretación}

De las encuestas realizadas de acuerdo a los resultados obtenidos de la pregunta el $78 \%$ se encuentra de acuerdo con que se mejoren los créditos productivos para estimular la gestión financiera de los clientes empresariales mientras que un porcentaje del $22 \%$ considera lo contrario.

Se debe considerar la implementación de un pan estratégico financiero en el Banco Pichincha para que se puedan tomar las decisiones pertinentes y cumplir de manera adecuada con las necesidades de los clientes empresariales.

\section{Razones Financieras}

El manejo eficaz de liquidez es una habilidad que tiene mucha más importancia a medida que la entidad cambia su estructura financiera de las aportaciones de asociados a los depósitos de ahorro, que son más volátiles. La liquidez se refiere al efectivo necesario para retiros, una variable que el Banco no puede controlar.

\section{Tabla 1: Razón de liquidez}

\begin{tabular}{ccc}
\hline Razón & Fórmula & Aplicación \\
\hline $\begin{array}{l}\text { Cobertura de } \\
\text { depositantes }\end{array}$ & Cobertura 25 mayores depositantes & $300,46 \%$ \\
& Cobertura 100 mayores depositantes & $228,59 \%$ \\
\hline
\end{tabular}

Fuente: Balances

Elaborado por: Los autores

El indicador de liquidez general del Banco representa un total de 22,40\%, generando un nivel alto de disponibilidad frente a los depósitos totales a corto plazo, lo que quiere decir que atiende a las cuentas del pasivo con mayor exigibilidad. Cuanto mayor sea el valor de esta razón, mayor será la capacidad del Banco de pagar sus deudas.

Con relación a la cobertura de los 25 y 100 mayores depositantes la entidad se encuentra representado por $300,46 \%$ y $228,50 \%$ respectivamente lo que indica que el Banco tiene una capacidad financiera de liquidez alta. 


\section{Tabla 2: Razón de solvencia}

\begin{tabular}{ccc}
\hline Razón & Fórmula & Aplicación \\
\hline Solvencia & $\boldsymbol{L G}=\frac{\text { Patrimonio }}{\text { Activos }}$ & $\boldsymbol{L G}=\frac{\mathbf{8 5 3 . 6 9 0 , 0 0}}{9.885 .050,00}=0.0863$ \\
\end{tabular}

Fuente: Balances

Elaborado por: Los autores

La solvencia de una institución se entiende como su capacidad de cubrir obligaciones, en el caso de las instituciones financieras como se refiere a las obligaciones con sus clientes o socios, con sus acreedores u otros generadores de pasivos. El indicador óptimo no debe ser menos del 9\%, el porcentaje de solvencia obtenido es de 8,63\% lo que indica que la institución no tiene la capacidad de cubrir las obligaciones debido a que se encuentra por debajo del porcentaje indicado.

\section{Tabla 3: Razón de Estructura y calidad de activos}

\begin{tabular}{lcc}
\hline \multicolumn{1}{c}{ Razón } & Fórmula & Aplicación \\
\hline Razón de estructura & $\boldsymbol{L G}=\frac{\text { Activos improductivos netos }}{\text { Total de activos }}$ & $\boldsymbol{L G}=\mathbf{1 5 , 8 0}$ \\
financiera eficaz & $\boldsymbol{L G}=\frac{\text { Activos productivos }}{\text { Total de activos }}$ & $\mathbf{L G}=84,20 \%$
\end{tabular}

Fuente: Balances

Elaborado por: Los autores

Las inversiones financieras miden el porcentaje del activo total invertido en inversiones a largo plazo, además de ser importantes en la determinación de ganancias. La meta debe ser $\leq 2 \%$, en este caso el porcentaje de las inversiones financieras es de 15,80\%.

Tabla 4: Razón de rentabilidad

\begin{tabular}{lcc}
\hline \multicolumn{1}{c}{ Razón } & Fórmula & Aplicación \\
\hline Rendimiento operativo sobre activos & $\boldsymbol{L G}=\frac{\text { Resultado del ejercicio }}{\text { Total de activos }}$ & $\boldsymbol{L G}=0,49 \%$ \\
ROA & $\mathbf{L G}=\frac{\text { Resultado del ejercicio }}{\text { Total de patrimonio }}$ & $\boldsymbol{L G}=5,60 \%$ \\
Rendimiento sobre patrimonio ROE &
\end{tabular}

Fuente: Balances

Elaborado por: Los autores 
El rendimiento del activo total nos indica que, por cada $\$ 100$ que se invierten en el activo total nos genera un rendimiento de $\$ 0,49$.

En el Rendimiento sobre patrimonio del banco se obtuvo 5,60\% un porcentaje ligeramente superior al nivel óptimo que no debe ser menor de 3,67 \% según las entidades de control. Esta razón mide el nivel de retorno generado por el patrimonio, mientras más alto sea este porcentaje es mejor.

\section{Propuesta de estrategias de cambio en políticas de crédito y captaciones}

Estrategia $\mathbf{N}^{\circ} 1$ : Optimizar la entrega y recuperación del crédito

- Actualizar las políticas de crédito.

- Desarrollar campañas sobre las características del crédito empresarial, como el monto máximo que se puede otorgar.

- Incentivar a la microempresa sobre las bondades del microcrédito y brindar facilidades para el otorgamiento de este.

- Diseñar un nuevo esquema de cobranzas.

- Presionar periódicamente sobre la recuperación de cartera.

- Desarrollar estímulos o incentivos para los buenos pagadores de crédito.

Estrategia $\mathbf{N}^{\circ}$ 2: Incrementar la cobertura de créditos empresariales

- Acudir a empresas para socializar los servicios y ofertar créditos.

- Identificar la demanda de créditos empresariales.

- Establecer metas de colocación.

- Establecer políticas de incentivos.

- Colocar el efectivo en operaciones de crédito.

- Llegar a aquellas empresas que tienen una mayor cobertura.

Estrategia $\mathbf{N}^{\circ}$ 3: Incrementar los depósitos a plazo Fijo

- Otorgar tasas pasivas competitivas.

- Identificar la necesidad de clientes de obtener un ingreso adicional por sus ahorros.

- Establecer metas de captación.

- Establecer políticas de incentivos dependiendo del tiempo y del monto.

Estrategia $\mathbf{N}^{\circ}$ 4: Fidelizar a los clientes actuales

- Mejorar la atención al cliente. 
- Brindar una adecuada bienvenida a los clientes.

- Crear un modelo de incentivos para los empleados.

- Incorporar un buzón de quejas.

- Establecer un plan de mejoras en función de las observaciones obtenidas.

Estrategia $\mathbf{N}^{\circ}$ 5: Mejorar los procesos internos para la entrega de créditos empresariales

- Optimizar el tiempo en cada uno de los procesos

- Determinar un tiempo para la entrega de créditos empresariales

- Establecer tiempos para cada uno de los procesos internos como el análisis de las solicitudes de crédito.

- Comunicar oportunamente al cliente el resultado del crédito solicitado

Considerando la propuesta de estrategias de cambio en las políticas de crédito y captaciones el objetivo es fortalecer las estrategias, a mediano plazo, los resultados se evidencian en los estados financieros proyectados observando una mejora en las utilidades.

Tabla 52: Balance general proyectado

\begin{tabular}{|l|r|r|}
\hline \multicolumn{2}{|c|}{ PROYECCIÓN BALANCE GNERAL } \\
\hline \multicolumn{2}{|c|}{ (en miles de dólares) } \\
\hline ACTIVO & $\mathbf{2 0 1 7}$ & $\mathbf{2 0 1 8}$ \\
\hline & $1.605 .768,00$ & $1.846 .633,20$ \\
\hline Fondos disponibles & 0 & 0,00 \\
\hline Operaciones interbancarias & $1.616 .003,00$ & $1.858 .403,45$ \\
\hline Inversiones & $5.914 .713,00$ & $6.801 .919,95$ \\
\hline Cartera de créditos & 175 & 201,25 \\
\hline Deudores por aceptaciones & $191.695,00$ & $220.449,25$ \\
\hline Cuentas por Cobrar & $24.641,00$ & $28.337,15$ \\
\hline Bienes realizables por arrendamiento & $153.210,00$ & $176.191,50$ \\
\hline Institución & $609.851,00$ & $701.328,65$ \\
\hline Propiedad y equipo & $79.615,00$ & $91.557,25$ \\
\hline Otros activos & $\mathbf{9 . 5 1 8 . 2 2 2 , 0 0}$ & $\mathbf{1 1 . 7 2 5 . 0 2 1 , 6 5}$ \\
\hline TOTAL, ACTIVO & & \\
\hline PASIVO & $7.839 .116,00$ & $9.014 .983,40$ \\
\hline Obligaciones con el público & $42.381,00$ & $48.738,15$ \\
\hline Obligaciones inmediatas & $1.819,00$ & $2.091,85$ \\
\hline Aceptación en circulación & $245.146,00$ & $281.917,90$ \\
\hline Cuentas por pagar & $237.054,00$ & $272.612,10$ \\
\hline Obligaciones financieras & 0 & 0,00 \\
\hline Valores en circulación & $159.575,00$ & $183.511,25$ \\
\hline Obligaciones convertibles en acciones y aportes & $79.615,00$ & $91.557,25$ \\
\hline Otros Pasivos & $\mathbf{8 . 6 0 4 . 7 0 6 , 0 0}$ & $\mathbf{9 . 8 9 5 . 4 1 1 , 9 0}$ \\
\hline TOTAL PASIVO & & \\
\hline
\end{tabular}


ISSN: 2602-8085

Vol. 3, N², p. 78-97, abril - junio, 2019

\begin{tabular}{|l|r|r|}
\hline PATRIMONIO & & \\
\hline Capital Social & 607 & 698,05 \\
\hline Prima o Descuento en colocación de acciones & 0 & $1.476,00$ \\
\hline Reservas & $216.081,00$ & $248.493,15$ \\
\hline Otros aportes patrimoniales & 0 & $1.474 .942,30$ \\
\hline Superávit por valuaciones & $57.766,00$ & $66.430,90$ \\
\hline Resultados & $32.669,00$ & $37.569,35$ \\
\hline PATRIMONIO & $\mathbf{9 1 3 . 5 1 6 , 0 0}$ & $\mathbf{1 . 8 2 9 . 6 0 9 , 7 5}$ \\
\hline TOTAL PASIVO MAS PATRIMONIO & $\mathbf{9 . 5 1 8 . 2 2 2 , 0 0}$ & $\mathbf{1 1 . 7 2 5 . 0 2 1 , 6 5}$ \\
\hline
\end{tabular}

Fuente: Banco en estudio

Elaborado por: Los autores

Uno de los indicadores que se analizaron y tomando en cuenta la proyección para el año siguiente es, la Rentabilidad teniendo un incremento para el año 2018 según la siguiente tabla:

Tabla 4: Razón de rentabilidad proyectada

\begin{tabular}{ccc}
\hline Razón & $\mathbf{2 0 1 7}$ & $\mathbf{2 0 1 8}$ \\
\hline ROA & $0,49 \%$ & $0,67 \%$ \\
ROE & $5,60 \%$ & $7,15 \%$ \\
\hline
\end{tabular}

Fuente: Balances

Elaborado por: Los autores

La generación operativa mejora por el crecimiento de la cartera alcanzado el segundo semestre del año anterior, el manejo del portafolio que optimizó su rendimiento y la gestión de pasivos que logró disminuir el costo del fondeo. Se mantiene niveles de eficiencia en el gasto, pero el crecimiento fuerte de las provisiones absorbió una parte importante del margen operativo. A pesar de lo cual el Margen operacional neto del Banco creció de manera importante, mientras que en el GFP el crecimiento de los gastos operativos es mayor y el MON disminuye en el período interanual.

Sin embargo, la política de aseguramiento de su cartera y la gestión de recuperaciones originaron ingresos no operativos que respaldaron el resultado final que creció significativamente. Los indicadores de rentabilidad final mejoran sustancialmente en relación con mar-2017, recuperando su posición de años anteriores.

Para el año 2018 se espera mejorar la eficiencia operacional como fruto del crecimiento de las colocaciones de cartera y la optimización de sus procesos como resultado de la reingeniería de procesos y de las inversiones tecnológicas realizadas. Además, la eficiencia mejorará por el cambio en la estructura administrativa. 
ROA, mide la rentabilidad de los activos cuando mayores son valores en este ratio, representan una mejor condición de la empresa, del año 2017 en 0,49\% y al 2018 al 0,67\%.

ROE, mide el rendimiento promedio del patrimonio invertido por los accionistas de la entidad financiera del año 2017 es de 5,60\% al 7,15\% al año 2018 llegando a la conclusión de que la Planeación Financiera y el cambio de las políticas de crédito y captaciones lograra que la rentabilidad del Banco se incremente y tenga una mayor utilidad liquida.

\section{Conclusiones.}

- El sector productivo en el país, en gran parte depende de la utilización de créditos para realizar una inversión, y hacer que la misma se incremente, la entidad financiera en la actualidad no está utilizando las estrategias correctas para estimular la gestión productiva y financiera de los clientes empresariales.

- Según las encuetas realizadas a los clientes, el 100\% de ellos están de acuerdo con la disminución de trámites para el acceso a los créditos, en especial las pequeñas y medianas empresas en su mayor parte requieren del acceso a los créditos para poder incrementar su capital de trabajo sin embargo la generación de muchos trámites son un obstáculo para el acceso al mismo.

- Con la aplicación del análisis financiero se pudo determinar la situación actual del Banco, con la finalidad de establecer estrategias que permitan la optimización de la cartera de créditos empresariales y microcréditos. Es así como la planeación financiera se constituye en una herramienta fundamental para la toma de decisiones financieras por parte de los directivos.

- El desarrollo de la propuesta está enmarcada en mejorar la rentabilidad medida a través del ROA y ROE, situación que según la planeación financiera aplicada, y el cambio de las políticas de crédito y captaciones propuestas, se cumple; al logar un incremento del ROA del 0,49\% en el año 2017 al 0,67\% en el año 2018, al igual que el ROE se incrementa del 5,60\% en el año 2017, al 7,15\% en el año 2018.

\section{Referencias bibliográficas.}

Azahar, S., \& Castillo, J. (2006). INVESTIGACION SOBRE LA DEMANDA DE CREDITO BANCARIO POR PARTE DE LAS PEQUEÑAS Y MEDIANAS EMPRESAS EN EL SALVADOR. Trabajo de Graduación, UNIVERSIDAD CENTROAMERICANA “JOSE SIMEON CAÑAS" , El Salvador. Recuperado el 3 de

julio

de

2017,

de 
http://www.uca.edu.sv/deptos/economia/media/archivo/bf3a02_tesisdemandadecredito bancarioapymes.pdf

Cordova, M. (2012). GESTION FINANCIERA. BOGOTA: Ecoe Ediciones.

Corporacion Universitaria del Caribe. (2014). DIAGNOSTICO FINANCIERO. SINCELEJO -COLOMBIA: CECAR.

Del Valle, E. E. (2008). Crédito y Cobranzas. Recuperado el 3 de julio de 2017, de http://fcasua.contad.unam.mx/apuntes/interiores/docs/98/opt/credito_cobranza.pdf

Diez de Castro, L., \& López, J. (2008). Dirección financiera. Madrid: Pearson Educación, S.A.

Endara, P. E. (2004). LAS INSTITUCIONES MICROFINANCIERAS COMO INSTRUMENTO PARA EL DESARROLLO DEL PAÍS. Tesis presentada como requisito para optar al Título de Máster, INSTITUTO DE ALTOS ESTUDIOS NACIONALES, Quito. Recuperado el 3 de julio de 2017, de http://repositorio.iaen.edu.ec/bitstream/24000/265/1/IAEN-007-2004.pdf

EOI Escuela de Negocios. (2010). PLANEAMIENTO ESTRATÉGICO FINANCIERO NOTA TÉCNICA. Recuperado el 4 de julio de 2017, de http://api.eoi.es/api_v1_dev.php/fedora/asset/eoi:48199/componente48197.pdf

Lawrence, J. (2013). Principios de Administrción Financiera. Mexico: Person Educación, S.A de C.V.

Medina , E., \& Montes, V. (2010). CRÉDITO Y COMERCIO INTERNACIONAL, PILARES DEL DESARROLLO EN EL MUNDO. Revista ICE(853), 5-24. Recuperado el 3 de julio de 2007, de CRÉDITO Y COMERCIO INTERNACIONAL, PILARES DEL DESARROLLO EN EL MUNDO: http://www.revistasice.com/CachePDF/ICE_853_5-

24_04D48EC2885CADA51E27703D5F854233.pdf

Ortega, A. (2012). Planeación Financiera Estratégica. México: McGraw-Hill.

Prieto, C. (2010). Análisis Financiero. Bogotá: Fundación para la Educación San Mateo.

Rodríguez, J. (2015). Impacto financiero causado por los cuentagotas en microempresarios de Villavicencio. Maestría en Administración, Villavicencio. Recuperado el 3 de julio de 2017, de Universidad Nacional de Colombia sede Manizales (UNILLANOS): http://www.bdigital.unal.edu.co/50446/1/17330041.2015.pdf

Solostocks. (2002). Guía para la venta de stocks. Recuperado el 3 de julio de 2017, de Capítulo 3: Impacto financiero en su empresa: http://www.mailxmail.com/curso-guia-ventastocks/impacto-financiero-empresa

SRI. (2017). ¿Quiénes son las personas naturales? Recuperado el 3 de julio de 2017, de http://www.sri.gob.ec/de/31

Universidad de Sonora. (2007). Capítulo I Antecedentes Históricos del Crédito. Recuperado el 3 de julio de 2017, de http://tesis.uson.mx/digital/tesis/docs/2437/Capitulo1.pdf

Valladolid Emprende. (2012). El diagnóstico financiero de la empresa: Manuales prácticos de gestión. Recuperado el 4 de Julio de 2017, de http://www.valladolidemprende.es/extras/EL_DIAGNOSTICO_FINANCIERO_DE_UNA_EMP RESA.pdf

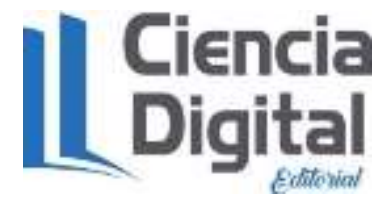




\section{PARA CITAR EL ARTÍCULO INDEXADO.}

Salazar M. , Alvear P. \& Sampedro M.. (2019). La planeación financiera como herramienta de gestión para mejorar la rentabilidad en las instituciones financieras., Revista electrónica Ciencia Digital 3(2), 78-97. Recuperado desde: http://cienciadigital.org/revistacienciadigital2/index.php/CienciaDigital/article/view/487/1126

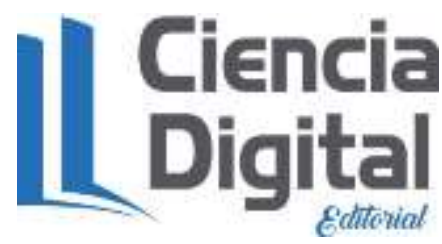

El artículo que se publica es de exclusiva responsabilidad de los autores y no necesariamente reflejan el pensamiento de la Revista Ciencia Digital.

El artículo queda en propiedad de la revista y, por tanto, su publicación parcial y/o total en otro medio tiene que ser autorizado por el director de la Revista Ciencia Digital.
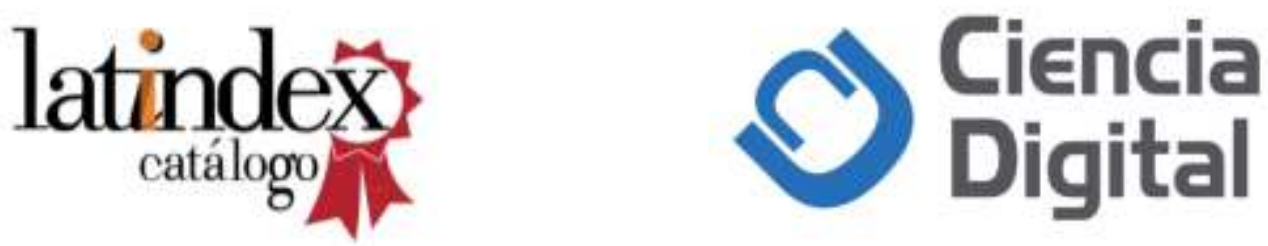